\title{
Endoscopic Retrograde Cholangiopancreatography (ERCP) in Cirrhotic Patients
}

\author{
Mohamed A Bassiony, Ahmed Omran \\ Gastroenterology and Hepatology Unit, Internal Medicine Department, \\ Faculty Of Medicine, Zagazig University, Egypt \\ See the article pages
}

\section{Introduction}

Endoscopic retrograde cholangiopancreatography (ERCP) is an endoscopic technique in which a specialized side-viewing upper endoscope is guided into the duodenum, the bile and pancreatic ducts are opacified by injection of a contrast medium, and allowing for a variety of therapeutic interventions [1].

Patients with cirrhosis can tolerate ERCP to treat their biliary tract or pancreatic diseases. Patients with liver cirrhosis are 3 times more susceptible to cholelithiasis, than the non-cirrhotic population plus the biliary and pancreatic cancer and other disease. Child-Pugh classification is the determinative factor of ERCP treatment and the most important predictor of outcome in these patients. The main severe complication postERCP is bleeding [2].

\section{Indications for ERCP}

Indications for ERCP in cirrhotic patients are no different from those in general population. According to National Institutes of Health statement in $2002[2,3]$ :

1. ERCP is sensitive and specific in diagnosis of choledocholithiasis.

2. ERCP with sphincterotomy and stone removal is a valuable therapeutic modality in choledocholithiasis with jaundice, dilated common bile duct, acute pancreatitis, or cholangitis.

3. In patients with pancreatic or biliary cancer, the principal advantage of ERCP is palliation of biliary obstruction when surgery is not elected.

4. Tissue sampling for patients with pancreatic or biliary cancer not undergoing surgery may be achieved by ERCP, but this is not always diagnostic.

5. ERCP is the best means to diagnose cancers of ampulla of vater.
6. In patients with severe biliary pancreatitis, early intervention with ERCP reduces morbidity and mortality compared with delayed ERCP.

7. ERCP with appropriate therapy is beneficial in selected patients who have either recurrent pancreatitis or pancreatic pseudocysts.

8. Patients with type I sphincter of Oddi dysfunction (SOD) respond to ERCP with sphincterotomy.

9. ERCP is the gold standard for diagnosis of primary biliary cirrhosis(PSC).

10. Diagnosis \& treatment of portal biliopathy that includes abnormalities in the intrahepatic and extrahepatic biliary tract, gallbladder and cystic duct secondary to portal hypertension mostly caused by distended venous collaterals .

\section{Contraindications for ERCP}

According to American Society of Gastrointestinal Endoscopy guidelines in 2005 [4]:

1- Diagnostic ERCP should not be undertaken in the evaluation of pancreaticobiliary pain in the absence of objective findings on other imaging studies.

2- Routine ERCP before laparoscopic cholecystectomy should not be performed.

\section{Other general contraindications [1]:}

Acute pancreatitis (unless persistently raised or worsening bilirubin suggests ongoing obstruction)

- Previous pancreatoduodenectomy.

- Marked coagulopathy if sphincterotomy planned.

- Recent myocardial infarction.

- Inadequate surgical back-up.

- History of contrast dye anaphylaxis.

- Poor health condition for surgery. 
- Severe cardiopulmonary disease. Complications of ERCP

According to American Society of Gastrointestinal Endoscopy guidelines in 2005 [4]:

ERCP is associated with some complications and adverse events can be divided into two main groups:

\section{1- Specific complications [6]:}

Pancreatitis: The most frequent complication of ERCP, although most cases are mild and pass without complications.

Bleeding: Bleeding during ERCP typically develops after sphincterotomy.This is the most common \& most serious in cirrhotic patients.

Infection: Infections occurring after ERCP are most often due to manipulation of an obstructed biliary or pancreatic system.

Perforation: ERCP may rarely be complicated by perforation of the esophagus, stomach, duodenum, or jejunum.

\section{2- General complications :}

Medication-related: complications related to Anticholinergic drugs, oversedation by benzodiazepines \&/or opiates, contrast allergy [6,7].

Cardiopulmonary complications: such as aspiration, hypoxemia, gas embolisms and cardiac dysrhythmia [8].

Electrosurgical hazards: Excessive cautery can lead to perforation, while inadequate cautery increases the risk of bleeding. [5,8].

Miscellaneous complications: include gallstone ileus, colonic perforation, liver abscess, splenic, hepatic or vascular trauma, pneumothorax, impaction of retrieval baskets, complications related to biliary and pancreatic stents, biloma [6].

In this issue of the Afro-Egyptian Journal of Infectious and Endemic diseases, El-Naggar et al., performed free hand needle fistulotomy in the subgroup of cirrhotic patients with difficult cannulation. The maneuver was performed with high success rate and fewer complications. These findings emphasis that invasive techniques can be performed safely and successfully in well prepared cirrhotic patients. Cirrhosis is a major health problem in the Egyptian community and cirrhotic patients are not infrequently referred to the ERCP due to a variety of causes. The major problems that may face patients with cirrhosis are related to the bleeding tendencies and problems of anesthesia. According to El-Naggar et al., both can be controlled by proper preparation using the fresh plasma, vitamin $\mathrm{K}$ supplementation as well as step wise use of sedation. This study open the doors for application of further invasive techniques in cirrhotic patients without fear from further complications. However, this study have some limitations. Firstly, the limited number of patients in each arm. Secondly, exclusion of patients with Child $\mathrm{C}$ cirrhosis, in fact these patients represent challenge for all invasive procedures and are frequently seen with indications for ERCP, probably further studies focusing these patients may answer the question whether or not Child $\mathrm{C}$ cirrhotics can tolerate ERCP.

\section{References}

1. Classen M, Tytgat GNJ, Lightdale CJ. Gastroenterological Endoscopy. New York: Thieme Publishing Group 2010:790.

2. Zhou Y-F, Zhang X, Zhang X-f, Guo Y LV Wen, Lin $X$ et al., ERCP in patients with liver cirrhosis : analysis of 156 cases . Chin J Hepatobiliary Surgery 2009;15 (9):647-650.

3. Cohen S, Bacon BR, Berlin JA, Fleischer D, Hecht GA, Loehrer PJ Sr. National Institutes of Health State-of-the-Science Conference Statement: ERCP for diagnosis and therapy, January 14-16, 2002. Gastrointest Endosc. 2002;56(6):803-9.

4. Adler DG, Baron TH, Davila RE, Egan J, Hirota WK, Leighton JA, et al. ASGE guideline: the role of ERCP in diseases of the biliary tract and the pancreas. Gastrointest Endosc. 2005;62(1):1-8.

5. Glomsaker TB, Hoff G, Kvaløy JT, Søreide K, Aabakken L, Søreide JA. Patient-reported outcome measures after endoscopic retrograde cholangiopancreatography: a prospective, multicentre study. Scand J Gastroenterol. Jul 2013;48(7):868-76.

6. Testoni PA, Mariani A, Giussani A, Vailati C, Masci E, Macarri G. Risk factors for post-ERCP pancreatitis in high- and low-volume centers and among expert and non-expert operators: a prospective multicenter study. Am $J$ Gastroenterol. 2010; 105(8):1753-6. 
7. Draganov PV, Forsmark CE. Prospective evaluation of adverse reactions to iodinecontaining contrast media after ERCP. Gastrointest Endosc. 2008;68(6):1098101.
8. Kwon CI, Song SH, Hahm KB, Ko KH. Unusual complications related to endoscopic retrograde cholangiopancreatography and its endoscopic treatment. Clin Endosc. 2013;46(3):251-9. 